\title{
RADAR CONTRIBUTIONS TO ASTEROID ASTROMETRY AND DYNAMICS
}

STEVEN J. OSTRO

Jet Propulsion Laboratory, California Institute of Technology Pasadena, California, USA

\section{Introduction}

Almost every radar observation of asteroids has measured some characteristics of the distribution of echo power in time delay (range) and Doppler frequency (radial velocity). Such measurements, which are orthogonal to optical plane-of-sky angular astrometry, are also done in the well-known, "absolute" reference frame of the planetary ephemerides, often with fractional precision that is much finer than can be achieved with other kinds of groundbased observations; they therefore can dramatically refine orbits and ephemerides. This statement is most true for near-Earth asteroids (NEAs), which constitute the most prolifically observed radar targets and are likely to be observed in increasing abundance as instrumental capabilities improve and CCD-based, NEA search programs expand. Moreover, studies of closely approaching NEAs benefit tremendously from the inverse fourthpower dependence of echo strength on target distance. For these reasons, I devote the bulk of this paper to NEAs.

\section{Systems and Techniques}

The Arecibo and Goldstone radar systems, the most sensitive on the planet, are responsible for the vast majority of radar observations reported to date, although radar astronomy has a long history in Russia and, during the past few years, two-station asteroid radar observations have used international combinations of antennas in Russia, Germany, Japan, and the United States. The upgraded Arecibo 305-m telescope, expected to become 
operational in late 1996 , will be nearly a factor of 40 more sensitive than the Goldstone 70-m antenna (DSS14), which in turn is many times more sensitive than other available radar systems. Arecibo will have twice the range and will see three times the volume of Goldstone, while Goldstone, which is fully steerable, will see twice the plane-of-sky solid angle and will cover three times more hour angle than Arecibo, which cannot point more than $\sim 20$ degrees from the zenith.

Whereas sensitivity is the primary factor that limits a radar system's capabilities in astrometry or imaging, it is not the only one. It turns out that two-station (bistatic) systems can have significant advantages over single-station (monostatic) systems in observations of very close, and/or very rapidly moving targets (NEAs). Full steerability is generally advantageous even for the most distant radar-detectable targets (in the Saturn system). Bistatic configurations using Goldstone's 70-m antenna to transmit and either a second Goldstone antenna (the 34-m DSS13) or the 27cantenna Very Large Array (VLA) in New Mexico to receive have made unique measurements. For example, interferometric observations with the G-VLA configuration can synthesize a beamwidth as small as 0 "'25 (vs. $2^{\prime}$ for single-dish observations), and is capable of $0 . \prime 01$ astrometry in the radio reference frame. Future bistatic experiments may link Arecibo or Goldstone to the 100-m Greenbank Telescope (GBT, under construction) or to each other.

The simplest radar experiments transmit a highly monochromatic, continuous-wave (cw) signal $(2380-\mathrm{MHz}, 13 \mathrm{~cm}$ for Arecibo; $8510 \mathrm{MHz}, 3.5 \mathrm{~cm}$ for Goldstone). One tunes the receiver continuously to the echo's Dopplershifted frequency using a prediction ephemeris calculated from an orbit solution that, at least initially, is based on optical astrometry only. A correction to the ephemeris is measured, and the resultant Doppler datum is used in a next-generation orbit solution, which yields a refined Doppler ephemeris. Similarly, "ranging" observations use time-modulated waveforms and measure corrections to time-delay predictions. (My 1993 review of planetary radar astronomy describes waveforms and observational techniques in more detail.)

To do any radar observations, one prefers to be able to point within $\sim 20^{\prime \prime}$ to avoid significant loss of echo power. One also prefers small Doppler uncertainties, so the receiver and data-acquisition system can use a narrow frequency window and hence a slow data-acquisition rate. Generally, one must integrate echoes to fight noise, so there are limits on how fast the echo can "drift through" the ephemeris before smear degrades echo strength (and resolution).

The rate of change of the delay-prediction error is the Doppler-prediction error times the negative reciprocal of the transmitter frequency. 
A Doppler error of $1 \mathrm{~Hz}$ (one-half wavelength per second) corresponds to a delay error rate of $\sim 36$ and $\sim 10$ microseconds per day at the Arecibo and Goldstone transmitter frequencies. In practice, one iterates, using increasingly fine-resolution delay-Doppler astrometry to produce increasingly accurate orbit solutions, aiming to freeze the target in the ephemeris.

\section{Astrometry}

In reporting radar astrometry, one specifies the reference points on the transmitting antenna, the receiving antenna, and the target. The target's center of mass (COM) is what matters for orbit estimations, but echoes are reflected from the surface, so eventually uncertainty about the target's size and shape may be the dominant source of astrometric error, and hence in the degree to which radar can refine an orbit estimate. Therefore, orbit refinement is tightly coupled to determination of physical properties. Radar measurements that produce new information about a target's size, shape, rotation, or surface properties also may provide useful astrometry, and vice versa. The relative utility of the different kinds of information provided depends on the caliber of the radar data and the available prior information, as well as on the sophistication of analysis techniques. Timeresolved measurements generally can achieve finer fractional precision than Doppler-only measurements and are thus more useful, but they also require stronger echoes.

Asteroid radar astrometry dates to 1968 , but nearly two decades passed before Yeomans et al. (1987) carried out the first systematic exploration of the utility of delay-Doppler data for the refinement of asteroid and comet orbits and prediction ephemerides. They demonstrated that a single radar detection can ensure the recovery of newly discovered asteroids, shrinking the instantaneous positional uncertainty by orders of magnitude with respect to an optical-only orbit. Even for an asteroid with an extensive optical-data history, a few radar measurements can shrink the positional error ellipsoid by a factor of 2 for at least a decade.

Useful Doppler astrometry has been obtained for all 38 radar-detected NEAs and time-delay astrometry has been obtained for 17 NEAs and 3 MBAs. All 1980-1990 delay/Doppler was reported by Ostro et al. 1991, who also tabulated all other asteroid and comet radar astrometry through 1990; Yeomans et al. (1992) estimated the orbits for each of those objects. Errors in optical-only ephemerides have been found to be as large as $\sim 100000 \mathrm{~km}$ for newly discovered NEAs, but rarely exceed several target radii for MBAs.

The following table conveys the state of the art for radar reconnaissance of a fairly large asteroid during a close Earth approach: 
TABLE 1. Asteroid radar measurement precision.

\begin{tabular}{rrr}
\hline & \multicolumn{1}{c}{$\begin{array}{c}\text { Range } \\
\text { [meters] }\end{array}$} & $\begin{array}{c}\text { Radial Velocity } \\
\text { [meters/sec] }\end{array}$ \\
\hline Best Radar Resolution & $\sim 10$ & $\sim 0.0001$ \\
Asteroid "size" & $\sim 1000$ & 0.01 to 1 \\
Asteroid "location" & $\sim 10000000000$ & 10000 \\
\hline
\end{tabular}

In principle, it is not hard to resolve such a target into 10000 imaging pixels. Given adequate orientational coverage, a sequence of delay-Doppler images can be inverted to produce a detailed physical model (Hudson, 1993) and simultaneously to locate the COM's position with an uncertainty that is comparable to or smaller than the delay-Doppler cell resolution. In other words, COM astrometry with sub-decameter accuracy is within the reach of modern radar capabilities. Let me try to communicate the progress and potential of asteroid radar astrometry by relating some of our experiences with NEAs.

\subsection{APOLLO}

The 1980 Arecibo observations of this object (Ostro et al., 1991) achieved the first ranging to an Earth-crosser, with difficulty. Shortly after the observations began, B. G. Marsden notified us via telephone that new optical astrometry had revealed our prediction ephemeris to be in error by several arcminutes. We implemented pointing corrections, and then saw echoes that were much stronger than in any previous asteroid radar experiment.

The available delay-Doppler resolution was limited by the data-acquisition hardware and software, letting us resolve the object into only 10 cells in Doppler and about 1.5 in delay. The initial delay corrections to original ephemeris were $\sim 21 \mathrm{~ms}$.

\subsection{CASTALIA (1989 PB)}

Castalia was discovered on August 9, 1989, by E. F. Helin. Two days later, orbital calculations showed that this object was approaching the Earth and would pass within the Arecibo Observatory's field of view during August 19-22 at distances likely to provide echoes much stronger than in previous asteroid radar observations, during time windows that had already been allocated for 12 Victoria. The asteroid's trajectory passed within $0.027 \mathrm{AU}$ (11 lunar distances) from Earth on August 25, when the Voyager 2 space- 
craft was making its closest approach to Neptune. (The Goldstone 70-meter antenna was occupied with Voyager communications during the Neptune encounter and was unavailable for radar astronomy until Aug. 30, when cw spectra were obtained.) More informative observations were conducted at Arecibo on August 19-22, thanks in part to follow-up astrometry by several dedicated optical astronomers, whose measurements during the previous week ensured that we would be able to point accurately. R. McNaught provided critical astrometry at Siding Spring despite the asteroid's proximity to the nearly full moon, and two observers in Great Britain (J. D. Shanklin at Cambridge and B. Manning at Stakenbridge) made useful measurements during the Aug. 17 total lunar eclipse.

The initial Arecibo observations disclosed ephemeris errors of about $200 \mathrm{~Hz}$ and $60 \mathrm{~ms}$ (1.5 Earth radii). By Aug. 22, J. F. Chandler at the Center for Astrophysics had used the radar astrometry to make an improved delay-Doppler ephemeris, which he sent to Arecibo via email. (Prior to email, this process would have relied on express airmail.) The improved ephemeris was used to make a 64-frame radar movie. Each frame placed no more than $\mathrm{a} \sim 30$ pixels on the asteroid, but the $150 \times \sim 170-\mathrm{m}$ resolution was adequate to reveal a consistently bimodal distribution of echo power, suggesting a bifurcated shape. Analysis of the data yielded astrometry with uncertainties set by the imaging resolution: 2 microseconds and $1 \mathrm{~Hz}$. The asteroid was recovered by McNaught in May 1990, 0.4 arcsec from the position predicted from radar+optical data and $\sim 24$ arcsec from that based on an optical prediction.

Reconstruction of Castalia's shape (HO94) established the asteroid's contact-binary shape and also produced refined COM astrometry with uncertainties of 0.3 microseconds and $0.1 \mathrm{~Hz}$. The current plane-of-sky and range uncertainties in Castalia's position during its next close approach (to $0.025 \mathrm{AU}$ in 2046) are about 3 arcmin and $2000 \mathrm{~km}$ (D. K. Yeomans, pers. comm.).

\subsection{TOUTATIS $(1989 \mathrm{AC}=1934 \mathrm{CT})$}

Toutatis was (re)discovered in early 1989, nearly four years before its approach to 0.024 AU on Dec. 8, 1992. Despite the long optical-astrometric time base (1934 to Nov. 1992), the initial radar measurements corrected the ephemerides by $2.5 \mathrm{~ms}$. After radar refinements in the orbit during the week before the closest approach, the radar corrections were all smaller than the asteroid's delay-Doppler dispersion (typically $\sim 2 \mathrm{~Hz}$ and $\sim 10$ microsecond); that is, uncertainty in astrometry tied to Toutatis' COM was due to uncertainty in the asteroid's size and shape. The images showed a bizarre, partially bifurcated object in a highly unusual, non-principal- 
axis (NPA) spin state with several-day characteristic time scales. Detailed definition of Toutatis' spin state (and shape) required inversion (Hudson, 1993) of the image sequence with a comprehensive physical model. Hudson and Ostro (1995) carried out such an inversion on a low-resolution (typically 0.5 -microsecond $\times 0.1-\mathrm{Hz}$ ) subset of the images, estimating the asteroid's shape and inertia tensor, their mutual orientation, initial conditions for the asteroid's spin and orientation, the radar scattering properties of the surface, and the COM's delay-Doppler trajectory. For example, for monostatic $8510-\mathrm{MHz}$ observations with DSS-14 on Dec. 8, the estimates of the time delay and Doppler frequency of echoes from Toutatis' COM received at 14:40:00 UTC are $24.202819270 \mathrm{sec} \pm 0.12$ microseconds and $-58768.687 \pm 0.02 \mathrm{~Hz}$. The radar+optical orbit solution, estimated with DE 245 and confirmed with PEP740, yielded weighted rms residuals of 0.83 arc seconds, $0.087 \mathrm{~Hz}(1.5 \mathrm{~mm} / \mathrm{s}$ in radial velocity) and 0.39 microseconds ( $59 \mathrm{~m}$ in range). This solution indicates that Toutatis will pass $0.010360 \mathrm{AU}$ (four lunar distances) from Earth on 29 September 2004, the closest approach predicted for any asteroid or comet between now and 2060 (Yeomans and Chodas, 1994).

Toutatis' dimensions along the principal axes are $1.92,2.40$, and $4.6 \mathrm{~km}$. It is rotating in a long-axis mode characterized by periods of 5.41 days (rotation about the long axis) and 7.35 days (average for long-axis precession about the angular momentum vector). The principal moments of inertia are in ratios within $3 \%$ of 3.19 and 3.01 , and the inertia tensor is indistinguishable from that of a homogeneous body. (Such information has yet to be determined for any other asteroid or comet, and probably is impossible to acquire in a fast spacecraft flyby.) At this writing, the highest-resolution $(0.125$-microsecond $\times 0.008-\mathrm{Hz})$ images are being incorporated into the inversion. It will be interesting to fit shape, spin-state, and orbital parameters simultaneously to images with decameter resolution.

\subsection{GEOGRAPHOS}

Geographos' Aug. 25, 1994, approach to $0.0333 \mathrm{AU}$, the closest for at least the next two centuries, provided a unique opportunity for Goldstone radar observations. This experiment was given special impetus by the Clementine mission, which planned a $10.7 \mathrm{~km} / \mathrm{s}$ flyby of Geographos on Aug. 31 at a miss distance of approximately $100 \mathrm{~km}$ (Nozette and Garrett, 1994; a computer malfunction led to cancellation of the flyby.). We intended to use radar astrometry to improve the pre-encounter ephemeris and to use imaging with complete rotational coverage to optimize the post-encounter physical model of the asteroid.

The asteroid entered Goldstone's declination window on Aug. 28 and Ostro et al. (1996, Icarus) observed it daily for a week, obtaining over 400 
images whose linear spatial resolutions range from $\sim 75 \mathrm{~m}$ to $\sim 151 \mathrm{~m}$, The images contain an intrinsic north/south ambiguity, but the equatorial view allowed accurate determination of the shape of the radar-facing part of the asteroid's pole-on silhouette at any rotation phase. Sums of co-registered images defined the extremely elongated shape of that silhouette (Ostro et al., 1995, Nature), revealed craters and other contrast features "inside" the silhouette, and also showed curious protuberances at the asteroid's ends that may be related to the pattern of ejecta removal and deposition caused by the asteroid's gravity field. The delay-Doppler trajectory of Geographos' center of mass was determined to about $200 \mathrm{~m}$ on Aug. 28 and to about $100 \mathrm{~m}$ on Aug. 31, an improvement of two orders of magnitude over preobservation ephemerides.

The Geographos experience suggested that with adequate radar reconnaissance, it would be possible for a spacecraft lacking onboard optical navigation to be guided into orbit around, or collision course with, an asteroid. If onboard opnav capability were available, radar would nonetheless shrink the navigation uncertainties significantly, perhaps reducing the missions fuel requirements enough to allow a larger mass budget for science instrumentation. For example, Goldstone observations would have shrunk the positional error ellipsoid of Geographos just prior to a Clementine flyby of that target on Aug. 31, 1994. Before the Goldstone observations, the ellipsoid's typical overall dimension was $\sim 11 \mathrm{~km}$. Ranging on Aug. 28-29 and a preliminary shape reconstruction collapsed the ellipsoid's size along the line of sight to several hundred meters, so its projection toward Clementine on its inbound leg would have been $11 \times 2 \mathrm{~km}$. Goldstone-VLA plane-ofsky astrometry could have shrunk the error ellipsoid's longest dimension to about $1 \mathrm{~km}$, about half of Geographos's shortest overall dimension.

\subsection{GOLEVKA (1991 JX)}

This asteroid was ranged with sub-microsecond time resolution at both Arecibo and Goldstone, shortly after Helin discovered it in 1991. It was recovered in March $1995,5^{\prime \prime}$ and $3600^{\prime \prime}$ from positions based on radar+optical and optical-only predictions (D. K. Yeomans, pers. comm.). By then, the cycle time for updating delay-Doppler prediction ephemerides had been shrunk dramatically by D. K. Yeomans and J. D. Giorgini's JPL On-site Orbit Determination Program (OSOD). Installed at Goldstone prior to the Geographos experiment, this user-friendly software package made it possible for us to use radar (or optical) astrometry to refine ephemerides at the telescope. In June 1995, we took advantage of OSOD during an ambitious series of Goldstone observations. During June 3-8, we proceeded through three generations of orbit solutions, until our only limitation was uncer- 
tainty in the location of the asteroid's COM. That is, our ephemeris was "inside" the asteroid. Fortunately (for astrometry but not for imaging), the asteroid is only several hundred meters across, so COM time-delay astrometry had an uncertainty of only 2 microseconds. By June 9 , when the asteroid made its closest approach $(0.034 \mathrm{AU})$, we were using a refined ephemeris that was later found to be accurate to $0.01 \mathrm{~Hz}(0.2 \mathrm{~mm} / \mathrm{s}$ in radial velocity). On two dates we conducted radar aperture-synthesis observations, with Goldstone transmitting and the VLA receiving; the resultant images yield angular positions with absolute uncertainties of a few hundredths of an arcsec (P. Palmer, pers. comm.). On June 13-15 we carried out the first intercontinental radar astronomy observations, consisting of Goldstone cw transmissions and reception of the asteroid's echoes with the Evpatoria (Ukraine) 70-m antenna on each of those dates and reception of echoes with the Kashima (Japan) 34-m antenna on June 15. The full 1991-1995 radar+optical astrometric data set allows an accurate orbit integration some seven centuries into the future (D. K. Yeomans, pers. comm.).

\section{6. $1996 \mathrm{JG}$}

This asteroid was discovered by R. McNaught on May 8. Ten minutes of observations on May 26 revealed a $580-\mathrm{Hz}(20 \mathrm{~m} / \mathrm{s})$ correction to the opticalonly ephemerides. On the next day's five-hour track, we did two iterations with OSOD, producing ephemerides whose delay-prediction errors decreased from $\sim 4600$ microseconds to $\sim 20$ microseconds.

\section{Expectations and Concluding Remarks}

At this writing, the upgraded Arecibo telescope appears to be within six months of first "radar light". The impact of this instrument on astrometric and physical studies of asteroids is likely to be far-reaching. During its first decade of operation, it should provide several-hundred-pixel images of $\sim 100 \mathrm{MBAs}$ and several-thousand-pixel images of $\sim 50 \mathrm{NEAs}$, and the best radar COM astrometry should be good to a few meters for NEAs and a few kilometers for MBAs. In the past, radar has barely skimmed the inner edge of the main belt, but the upgraded Arecibo will have access to asteroids throughout the belt. The instrument should be technically able to do ranging to a an average of one MBA per week. Short-period comets, which generally lie at the edge of the current detectability window, will become easier targets, although NEA opportunities will be one to two orders of magnitude more frequent.

Apart from astrometry, asteroid radar is contributing to other arenas within dynamics through the production of the first detailed shape models of 
small NEAs. The Castalia and Toutatis models have been used by Scheeres et al. (1996 and 1995, respectively) to study close orbits around those bodies. Our goal is to explore the short-term evolution and long-term stability of those orbits, with application to robotic or piloted spacecraft, natural satellites, and non- escaping impact ejecta. Almost all these calculations rest on a gravity field calculated from a closed-form expression (Werner, 1994) for a polygonal representation of the shape under the assumption of uniform density. Stable and unstable periodic orbits exist about each body, and the fate of material launched from the surface is a strong function of initial velocity and surface location.

The Castalia model also is currently being used by E. Asphaug et al. (1996) to simulate the effects of impacts in various energy regimes; since one of those effects is to launch material into return orbits, the simulations provide initial conditions for dynamical investigations of many-particle systems in a novel dynamical regime.

Acknowledgements. This research was conducted at the Jet Propulsion Laboratory, California Institute of Technology, under contract with the National Aeronautics and Space Administration.

\section{References}

Asphaug, E., Ostro, S.J., de Jong, E.M., Scheeres, D.J., Suzuki, S., Hudson, R.S., and Benz, W.: 1996, "Disruptive impacts into an Earth-approaching asteroid", Bull. Am. Astron. Soc., in press.

de Pater, I., Palmer, P., Mitchell, D.L., Ostro, S.J., Yeomans, D.K., and Snyder, L.E.: 1994, "Radar aperture synthesis observations of asteroids", Icarus 111, 489-502.

Hudson, R.S.: 1993, "Three-dimensional reconstruction of asteroids from radar observations", Remote Sensing Reviews 8, 195-203.

Hudson, R.S. and Ostro, S.J.: 1994, "Shape of asteroid 4769 Castalia (1989 PB) from inversion of radar images", Science 263, 940-943.

Hudson, R.S. and Ostro S.J.: 1995, "Shape and non-principal axis spin state of asteroid 4179 Toutatis", Science 270, 84-86.

Hudson, R.S. and Ostro, S.J.: 1995, "Radar-based physical models of Earth-crossing asteroids", Bull. Am. Astron. Soc. 27, 1062.

Ostro, S.J.: 1993, "Planetary radar astronomy", Rev. Mod. Phys. 65, 1235-1279.

Ostro, S.J.: 1994, "The role of groundbased radar in near-Earth object hazard identification and mitigation", in: Hazards Due to Comets and Asteroids (T. Gehrels, M.S. Matthews, eds), Univ. of Arizona Press, 259-282.

Ostro, S.J., Campbell, D.B., Chandler, J.F., Shapiro, I.I., Hine, A.A., Velez, R., Jurgens, R.F., Rosema, K.D., Winkler, R., and Yeomans, D.K: 1991, "Asteroid radar astrometry", Astron. J. 102, 1490-1502.

Ostro, S.J., Hudson, R.S., Jurgens, R.F., Rosema, K.D., Winkler, R., Howard, D., Rose, R., Slade, M.A., Yeomans, D.K., Giorgini, J.D., Campbell, D.B., Perillat, P., Chandler, J.F., and Shapiro I.I.: 1995, "Radar images of asteroid 4179 Toutatis", Science $270,80-83$.

Ostro, S.J., Rosema, K.D., Hudson, R.S., Jurgens, R.F., Giorgini, J.D., Winkler, R., Yeomans, D.K., Choate, D., Rose, R., Slade, M.A., Howard, S.D., and Mitchell D.L.: 1995, "Extreme elongation of asteroid 1620 Geographos from radar images", Nature 375, 474-477. 
Ostro, S.J., Choate, D., Cormier, R.A., Franck, C.R., Frye, R., Giorgini, J., Howard, D., Jurgens, R.F., Littlefair, R., Mitchell, D.L., Rose, R., Rosema, K.D., Slade, M.A., Strobert, D.R., Winkler, R., Yeomans, D.K., Hudson, R.S., Palmer, P., Snyder, L.E., Zaitsev, A., Ignatov, S., Koyama, Y., and Nakamura A.: 1995, "Asteroid 1991 JX: The 1995 Goldstone radar experiment", Bull. Am. Astron. Soc. 27, 1063.

Ostro, S.J., Jurgens, R.F., Rosema, K.D., Hudson, R.S., Giorgini, J.D., Winkler, R., Yeomans, D.K., Scheeres, D.J., Choate, D., Rose, R., Slade, M.A., Howard, S.D., and Mitchell, D.L.: 1996, "Radar images of Geographos", Icarus 121, 44-66 .

Scheeres, D.J., Ostro, S.J., Hudson R.S., and Werner R.A.: 1996, "Orbits close to asteroid 4769 Castalia", Icarus 121, 67-87.

Werner, R.A.: 1994, "The gravitational potential of a homogeneous polyhedron", Celest. Mech. \& Dyn. Astron. 59, 253-278.

Yeomans, D.K., Ostro, S.J., and Chodas P.W.: 1987, "Radar astrometry of near-Earth asteroids", Astron. J. 94, 189-200.

Yeomans, D.K., Chodas, P.W., Keesey, M.S., Ostro, S.J., Chandler J.F., and Shapiro, I.I.: 1992, "Asteroid and comet orbits using radar data", Astron. J. 103, 303-317.

Yeomans, D.K. and Chodas P.W.: 1994, "Predicting close approaches of asteroids and comets to Earth", in: Hazards Due to Comets and Asteroids (T. Gehrels, M. S. Matthews, eds), Univ. of Arizona Press, 241-258. 